KYUSHU-HET-11

SAGA-HE-49

Nov., 1993

\title{
Complex wave function, Chiral spin order parameter and

\author{
Phase Problem
}

\author{
Masahiro IMACHI* \\ and \\ Hiroshi YONEYAMA ${ }^{\dagger} * *$ \\ Department of Physics, Kyushu University, Fukuoka 812, Japan \\ and \\ Department of Physics, Saga University, Saga 840, Japan ${ }^{\dagger)}$
}

\footnotetext{
* e-mail:a71355a@kyu-cc.cc.kyushu-u.ac.jp

** e-mail:yoneyama@math.ms.saga-u.ac.jp
} 


\section{ABSTRACT}

We study the two dimensional Hubbard model by use of the ground state algorithm in the Monte Carlo simulation. We employ complex wave functions as trial function in order to have a close look at properties such as chiral spin order $(\chi \mathrm{SO})$ and flux phase. For half filling, a particle-hole transformation leads to sum rules with respect to the Green's functions for a certain choice of a set of wave functions. It is then analytically shown that the sum rules lead to the absence of the $\chi \mathrm{SO}$. Upon doping, we are confronted with the sign problem, which in our case appears as a "phase problem" due to the phase of the Monte Carlo weights. The average of the phase shows an exponential decay as a function of inverse temperature similarly to that of sign by Loh Jr. et. al. . We compare the numerical results with those of exact numerical calculations. 


\section{$\S 1$. Introduction}

The two dimensional Hubbard model has recently attracted much attention in view of possibly providing insight into the high- $T_{c} \mathrm{Cu}-\mathrm{O}$ superconductors. In the strong $U$ (Coulomb repulsive force) limit at half filling, the model is equivalent to the antiferromagnetic Heisenberg model, and in good agreement with the antiferromagnetic behavior of superconducting materials. When the system is doped, however, the ground state properties appear less clear. Concerning ground states of the strongly correlated electronic systems, various hypothetical states such as spin-liquid, ${ }^{1)}$ resonating valence bond( RVB) ${ }_{,}^{2,3)}$ and flux phase, etc. are proposed. Of particular interest is a possibility of the violation of $\mathrm{P}$ (parity) and $\mathrm{T}$ (time) invariance in such systems. ${ }^{1)}$ From the field theoretical point of view also, the relevance of spontaneous breakdown of such symmetries have been pointed out, and it is conjectured that a Chern-Simons term in $2+1$ dimensional

$\mathrm{QED},{ }^{5-7)}$ which is regarded as an effective theory describing the RVB picture, may violate the confinement of the fundamental charge ( the charge-spin separation). ${ }^{8}$ In spite of such intriguing arguments, however, any clear evidence has not so far been found.

The above ideas are proposed based basically upon the mean field approach, and therefore the stability of its vacuum against full quantum fluctuations is not clear at all. In such situations numerical approach is a suitable method to study the strongly correlated electron systems. Among various approaches, Monte Carlo simulation is one of the most promising methods. 
In the present paper we present a Monte Carlo study ${ }^{9,10)}$ of the two dimensional Hubbard model. ${ }^{11)}$ So far similar works have been done. Although our approach is basically the same, and the size of lattice is also quite limited, our motivation of the study is two fold. One is to use complex wave functions as a trial function in the ground state algorithm in order to have a close look at the properties stated above, namely, ones of the chiral spin order $(\chi \mathrm{SO})$ and flux phase, ${ }^{4,12)}$ where the complexity of the expectation values is involved. At halffilling, a particle-hole symmetry makes the theory transparent to look at. By use of such symmetry, for some choice of a set of wave functions (we call it "dual choice"), it is shown that there hold sum rules with respect to the Green's function with spin up and that with down. They are of different form for even and odd lattice spacings. We analytically show that these sum rules lead to the absence of the expectation value of the $\chi \mathrm{SO}$ parameter at half-filling. ${ }^{13)}$

Another motivation is to study an effect of such wave functions to the so called sign problem, which frequently arises in the quantum Monte Carlo simulations. A widely used algorithm employs the Hubbard-Stratonovich transformation, 9,10 ) rewriting the system to the $2+1$ dimensional classical system of the Ising dynamical variable. The conventional importance sampling with configurations of this variable encounters the negative weight which is originated from the fermionic determinant. This prevents probabilistic algorithm from being applied to the system. A way to circumvent the problem is to use the positive weight and to estimate expectation value of operator combined with the average value of the sign. This however causes large statistical errors for some cases. 
The origin of the sign problem in the framework of trial function approach is not yet clear, and thus a way to overcome it has not yet been established. Some improvements have been tried by employing optimized wave function. ${ }^{14)}$ The results depend very much on the choice of the wave functions of the trial state. It is therefore not trivial at all what to obtain if we employ the complex wave function. In our case the sign of the weights are replaced by their phase ("phase problem") . We paid a special attention to the average of the phase. In the present paper, we study, as doped cases, 14 fermion and 10 fermion systems on $4 \times 4$ lattice. The latter case is almost free from the phase problem, but the former receives rather serious effect. A measurement of the $\chi \mathrm{SO}$ for the former case unfortunately gets so large noises to extrapolate to the low temperature limit. However other physical quantities such as one particle density operator and spinspin correlations are rather in good agreement with the result obtained by exact numerical calculations. ${ }^{15-17)}$

This paper is organized as follows. In the following section, we present the formalism. In section 3, the half filled case is analytically discussed based upon a particle-hole transformation. Some choice of a set of wave functions is shown to lead to a sum rule. The absence of the expectation value of $\chi \mathrm{SO}$ is derived at half filling. In section 4, results of numerical simulations are presented for half filled and doped cases. Our conclusions and discussion are presented in section 5 . 


\section{$\S 2$ Hubbard model and Monte Carlo algorithm}

In this section, we shortly present the formalism for fixing notations as well as for making the paper self-contained. It is basically based on that of Hirsch ${ }^{9,18-20)}$ and Imada-Hatsugai. ${ }^{10)}$

The Hubbard hamiltonian is defined as

$$
H=-t \sum_{\langle i j\rangle \sigma} c_{i \sigma}^{\dagger} c_{j \sigma}+\text { h.c. }+U \sum_{i} n_{i \uparrow} n_{i \downarrow}
$$

where $c_{i \sigma}\left(c_{i \sigma}^{\dagger}\right)$ is an annihilation (creation) operator of an electron with spin $\sigma$ ( $\sigma=$ up or down) at site $i$, and $n_{i \sigma}$ is the number operator at site $i$ of spin $\sigma$. The first term is the hopping term of electron over nearest neighbor sites $\langle i j\rangle$, and the second stands for the on-site repulsive Coulomb potential with the strength $U(>0)$. An algorithm to perform Monte Carlo simulations here is so called ground state algorithm for fixed number of fermions. Expectation value of a physical observable $O$ is calculated by

$$
\langle O\rangle=\frac{\langle\Phi|O \exp (-\beta H)| \Phi\rangle}{\rho(\beta ; \Phi)}
$$

where

$$
\rho(\beta ; \Phi) \equiv\langle\Phi|\exp (-\beta H)| \Phi\rangle
$$

is given in terms of an appropriate trial state vector $|\Phi\rangle$. As $\beta$ goes to infinity, (2.2) gives an expectation value for true ground state $\left|\psi_{0}\right\rangle$, unless the trial function is orthogonal to the true ground state;

$$
\exp (-\beta H)|\Phi\rangle \rightarrow \exp \left(-\beta E_{0}\right)\left|\psi_{0}\right\rangle\left\langle\psi_{0} \mid \Phi\right\rangle
$$


For evaluating (2.2) we make Monte Carlo simulations. Introducing discrete pseudotime with interval $\Delta \tau, \rho(\beta ; \Phi)$ is rewritten as

$$
\rho(\beta ; \Phi)=\left\langle\Phi\left|(\exp (-\Delta \tau H))^{L}\right| \Phi\right\rangle
$$

where $\Delta \tau=\beta / L$ and $L$ denotes the Trotter size, i.e., the number of time slices. Separating the hopping term $H_{0}$ and the self-interaction term $H_{1}$ of the hamiltonian, one introduces Trotter-Suzuki formula, ${ }^{21,22)}$

$$
\exp (-\Delta \tau H)=\exp \left(\frac{-\Delta \tau}{2} H_{0}\right) \exp \left(-\Delta \tau H_{1}\right) \exp \left(\frac{-\Delta \tau}{2} H_{0}\right)+O\left((\Delta \tau)^{3}\right)
$$

To deal with the quartic interaction term $H_{1}$ of the fermion field, one introduces the Hubbard-Stratonovich (H-S) transformation of the Ising type for each site.

$$
\exp \left(-c n_{\uparrow} n_{\downarrow}\right)=\frac{1}{2} \sum_{s= \pm 1} \exp \left(2 a s\left(n_{\uparrow}-n_{\downarrow}\right)-\frac{c}{2}\left(n_{\uparrow}+n_{\downarrow}\right)\right)
$$

where $a=\tanh ^{-1} \sqrt{\tanh (c / 4)}$ for a constant $c$. Eqs.(2.5), (2.6) and (2.7) lead to

$$
\rho(\beta ; \Phi)=\sum_{\left\{s_{i l}\right\}}\left\langle\Phi\left|\prod_{l=1}^{L}\left(w_{0} w_{1}\left(\left\{s_{i l}\right\}\right) w_{0}\right)\right| \Phi\right\rangle
$$

where the summation is taken over the $\mathrm{H}-\mathrm{S}$ variable $s_{i l}$ on the $2+1$ dimensional lattice. Quantities $w_{0}$ and $w_{1}\left(\left\{s_{i l}\right\}\right)$ are defined as

$$
\begin{aligned}
w_{0} & =\exp \left(-\Delta \tau H_{0} / 2\right) \\
w_{1}\left(\left\{s_{i l}\right\}\right) & =\prod_{i=1}^{N}\left(\frac{1}{2} \exp \left[2 a_{U} s_{i l}\left(n_{\uparrow}-n_{\downarrow}\right)-\frac{\Delta \tau U}{2}\left(n_{\uparrow}+n_{\downarrow}\right)\right]\right)
\end{aligned}
$$

where $a_{U}=\tanh ^{-1} \sqrt{\tanh (\Delta \tau U / 4)}$, and $N$ is the number of lattice sites. 
We assume a factorization of spin up and down sectors for the trial state,

$$
|\Phi\rangle=\left|\Phi_{\uparrow}\right\rangle\left|\Phi_{\downarrow}\right\rangle
$$

and take wave functions of single particle basis for $M_{f}$ fermions for each spin sector

$$
\left|\Phi_{\sigma}\right\rangle=\prod_{m}^{M_{f}}\left(\sum_{i}^{N}\left(\phi_{\sigma}\right)_{m i} c_{i \sigma}^{\dagger}\right)|0\rangle
$$

where $\left(\phi_{\sigma}\right)_{m i}\left(i=1, \ldots, N ; m=1, \ldots, M_{f}\right)$ is a $M_{f} \times N$ matrix. In this paper we allow $\left(\phi_{\sigma}\right)_{m i}$ to be complex in general. Using $(2.11), \rho(\beta ; \Phi)(2.8)$, is explicitly represented in a matrix form

$$
\begin{aligned}
\rho(\beta ; \Phi) & =\sum_{\left\{s_{i l}\right\}} W(\beta ; s) \\
W(\beta ; s) & =W_{\uparrow}(\beta ; s) W_{\downarrow}(\beta ; s)
\end{aligned}
$$

where $W_{\sigma}(\beta ; s)$ is given by a determinant of a product of matrices

$$
W_{\sigma}(\beta ; s)=\operatorname{det}\left[\phi_{\sigma}^{\dagger} \prod_{l}^{L}\left(M_{0} M_{1 \sigma}(l) M_{0}\right) \phi_{\sigma}\right]
$$

Matrix $M_{0}$ is given by

$$
\begin{aligned}
& M_{0}=\exp (-K) \\
& K_{i j}= \begin{cases}-\Delta \tau t / 2 & \text { nearest neighbor }(\mathrm{i}, \mathrm{j}) \\
0 & \text { otherwise }\end{cases}
\end{aligned}
$$

while $M_{1}$, which depends upon the H-S variable, is a diagonal matrix with element

$$
\left(M_{1 \sigma}(l)\right)_{i i}=\exp \left[\sigma a_{U} s_{i l}-\Delta \tau / 2\right]
$$

for $\sigma=+1(-1)$ for spin up (down). 
According to Imada-Hatsugai, ${ }^{10)}$ single particle Green's function at the time slice $l$

$$
\left(G_{\sigma}(l)\right)_{i j} \equiv \frac{\left\langle L_{\sigma}(l)\left|c_{j \sigma}^{\dagger} c_{i \sigma}\right| R_{\sigma}(l)\right\rangle}{\left\langle L_{\sigma}(l) \mid R_{\sigma}(l)\right\rangle}
$$

is expressed as

$$
G_{\sigma}(l)=R_{\sigma}(l) g_{\sigma}(l) L_{\sigma}^{\dagger}(l)
$$

where

$$
g_{\sigma}(l)=\left(L_{\sigma}^{\dagger}(l) R_{\sigma}(l)\right)^{-1}
$$

Matrix $R_{\sigma}(l)$ is $N \times M_{f}$ matrix, and $L_{\sigma}^{\dagger}(l)$ is $M_{f} \times N$ one;

$$
\begin{aligned}
& R_{\sigma}(l)=M_{1 \sigma}(l) M_{0} \prod_{l=l+1}^{L}\left(M_{0} M_{1 \sigma}(l) M_{0}\right) \phi_{\sigma} \\
& L_{\sigma}^{\dagger}(l)=\phi_{\sigma}^{\dagger} \prod_{l=1}^{l-1}\left(M_{0} M_{1 \sigma}(l) M_{0}\right) M_{0}
\end{aligned}
$$

Finally, expectation value of operator with spin $\sigma$ for the ground state is averaged at each time slice, and given by

$$
\begin{aligned}
\left\langle O_{\sigma}\right\rangle & =\frac{\frac{1}{L} \sum_{l} \sum_{\{s\}} \operatorname{det}\left(L_{\sigma}(l)^{\dagger} O_{\sigma} R_{\sigma}(l)\right) W_{-\sigma}}{\sum_{\{s\}} W} \\
& =\frac{1}{L} \sum_{l\{s\}} \frac{\operatorname{det}\left(L_{\sigma}(l)^{\dagger} O_{\sigma} R_{\sigma}(l)\right)}{W_{\sigma}} W \frac{1}{\sum_{\{s\}} W}
\end{aligned}
$$

We employ the heat-bath method for updating the $\mathrm{H}-\mathrm{S}$ variables. $W$, however, may not be real in some cases, though $\rho(\beta ; \Phi)$ is positive semi-definite. These cases lead one to so called sign problem. In our case it is replaced by that of the phase, which prevents us from doing importance sampling. Conventional way out of it is to define a positive measure

$$
P_{+}=\frac{|W|}{\sum_{s}|W|}
$$


and evaluate (2.20) in such a way as

$$
\begin{aligned}
\left\langle O_{\sigma}\right\rangle & =\left(\frac{1}{L} \sum_{l\{s\}} \frac{\operatorname{det}\left(L_{\sigma}(l)^{\dagger} O_{\sigma} R_{\sigma}(l)\right)}{W_{\sigma}}|W| e^{i \theta_{w}}\right) \frac{1}{\sum_{\{s\}}|W| e^{i \theta_{w}}} \\
& =\left(\frac{1}{L} \sum_{l\{s\}} \frac{\operatorname{det}\left(L_{\sigma}(l)^{\dagger} O_{\sigma} R_{\sigma}(l)\right)}{W_{\sigma}}|W| e^{i \theta_{w}} / \sum_{\{s\}}|W|\right)\left(\frac{1}{\sum_{\{s\}}|W| e^{i \theta_{w}} / \sum_{\{s\}}|W|}\right) \\
& =\left\langle\frac{1}{L} \sum_{l} \frac{\operatorname{det}\left(L_{\sigma}(l)^{\dagger} O_{\sigma} R_{\sigma}(l)\right)}{W_{\sigma}} e^{i \theta_{w}}\right\rangle_{+} /\left\langle e^{i \theta_{w}}\right\rangle_{+}
\end{aligned}
$$

where $W \equiv|W| e^{i \theta_{w}}$, and $\langle\bullet\rangle_{+}$is the expectation value of $\bullet$ with the positive measure $P_{+}$.

\section{$\S 3$ Dual choice of wave function and sum rule}

The hamiltonian of the system respects a symmetry under particle-hole transformation

$$
c_{i \sigma}^{\dagger} \rightarrow \eta^{i} d_{i-\sigma}
$$

where $d_{i-\sigma}$ denotes the annihilation operator of a hole at site $i$ with spin $-\sigma$. The sign factor $\eta^{i}=(-1)^{i}$ is positive (negative) for even (odd) sites. In this section we will show the Green's functions satisfy sum rules when the system is half-filled, and "dual choice" of the wave function is made. The sum rule is satisfied when the particle-hole symmetry holds. The sum rules are

$$
f_{i j}= \begin{cases}\delta_{i j}-g_{j i}^{*} & \text { for }|i-j|=\text { even } \\ g_{j i}^{*} & \text { for }|i-j|=\text { odd }\end{cases}
$$

where $f_{i j}$ and $g_{i j}$ denote the spin up and down Green's function in the configuration space, respectively, and $*$ is the complex conjugation.

\section{3-1 particle-hole transformation}


Particle-hole ( $\mathrm{p}-\mathrm{h})$ transformation gives the correspondence

$$
|0\rangle \longleftrightarrow|\tilde{2}\rangle \quad|1 \sigma\rangle \longleftrightarrow|\tilde{1} \sigma\rangle \quad|2\rangle \longleftrightarrow|\tilde{0}\rangle
$$

at each site, where $|0\rangle,|1 \sigma\rangle$ and $|2\rangle$ denotes zero, single particle with spin $\sigma$ and two particles (spin up and down), while $|\tilde{0}\rangle,|\tilde{1} \sigma\rangle$ and $|\tilde{2}\rangle$ denotes zero hole, single hole with spin $\sigma$ and two holes, respectively. Note that hole states are denoted with tilde.

The creation operator of a particle with quantum number $m$ (e.g.,momentum) is given as

$$
c_{m \sigma}^{\dagger}=\sum_{j} \phi_{m j} c_{j \sigma}^{\dagger}
$$

where $\phi_{m j}$ is the wave function. Suffices $m$ and $j$ are for example abbreviated as $m=k=\left(k_{1}, k_{2}\right), j=n=\left(n_{1}, n_{2}\right)$ for the momentum $\left(k_{1}, k_{2}\right)$ and the position $\left(n_{1}, n_{2}\right)$. Let us see how states transform under the p-h transformation. For illustration we consider one-dimensional half-filled $\left(M_{f}=2\right)$ system with $N=4$ (4 site system). Spin $\sigma$ state is

$$
c_{m_{1} \sigma}^{\dagger} c_{m_{2} \sigma}^{\dagger}|0\rangle=\sum_{j_{1} j_{2}} \phi_{m_{1} j_{1}} \phi_{m_{2} j_{2}} c_{j_{1} \sigma}^{\dagger} c_{j_{2} \sigma}^{\dagger}|0\rangle
$$

where $m_{1}$ and $m_{2}$ are quantum numbers of the two particles with spin $\sigma$. Under the $\mathrm{p}-\mathrm{h}$ transformation, this state goes to

$$
\sum_{j_{1} j_{2}} \phi_{m_{1} j_{1}} \phi_{m_{2} j_{2}} \eta_{j_{1}} \eta_{j_{2}} d_{j_{1}-\sigma} d_{j_{2}-\sigma} d_{1-\sigma}^{\dagger} d_{2-\sigma}^{\dagger} d_{3-\sigma}^{\dagger} d_{4-\sigma}^{\dagger} d_{1 \sigma}^{\dagger} d_{2 \sigma}^{\dagger} d_{3 \sigma}^{\dagger} d_{4 \sigma}^{\dagger}|\tilde{0}\rangle
$$

We pay an attention to spin $-\sigma$ quantities, and then rewrite

$$
d_{1-\sigma}^{\dagger} d_{2-\sigma}^{\dagger} d_{3-\sigma}^{\dagger} d_{4-\sigma}^{\dagger}=\frac{1}{4 !} \sum_{l_{1} l_{2} l_{3} l_{4}} \varepsilon_{l_{1} l_{2} l_{3} l_{4}} d_{l_{1}-\sigma}^{\dagger} d_{l_{2}-\sigma}^{\dagger} d_{l_{3}-\sigma}^{\dagger} d_{l_{4}-\sigma}^{\dagger}
$$


where $l_{1}, . ., l_{4}$ denote the sites and $\varepsilon_{l_{1} l_{2} l_{3} l_{4}}$ stands for the Levi-Civita symbol. The quantities with $-\sigma$ in $(3.5)$ is

$$
\begin{aligned}
\sum_{j_{1} j_{2} l_{1} l_{2} l_{3} l_{4}} & \frac{6}{4 !} \phi_{m_{1} j_{1}} \phi_{m_{2} j_{2}} \eta_{j_{1}} \eta_{j_{2}} \varepsilon_{l_{1} l_{2} l_{3} l_{4}}\left(\delta_{j_{2} l_{1}} \delta_{j_{1} l_{2}}-\delta_{j_{2} l_{2}} \delta_{j_{1} l_{1}}\right) d_{l_{3}-\sigma}^{\dagger} d_{l_{4}-\sigma}^{\dagger}|\tilde{0}\rangle \\
= & -\frac{12}{4 !} \sum_{l_{1} l_{2} l_{3} l_{4}} \phi_{m_{1} l_{1}} \phi_{m_{2} l_{2}} \eta_{l_{1}} \eta_{l_{2}} \varepsilon_{l_{1} l_{2} l_{3} l_{4}} d_{l_{3}-\sigma}^{\dagger} d_{l_{4}-\sigma}^{\dagger}|\tilde{0}\rangle \\
= & -\frac{1}{2} \Delta \sum_{j_{3} j_{4} m_{3} m_{4}} \phi_{m_{3} j_{3}}^{*} \phi_{m_{4} j_{4}}^{*} \eta_{j_{3}} \eta_{j_{4}} \varepsilon_{m_{1} m_{2} m_{3} m_{4}} d_{j_{3}-\sigma}^{\dagger} d_{j_{4}-\sigma}^{\dagger}|\tilde{0}\rangle
\end{aligned}
$$

where $\Delta=\operatorname{det} \tilde{\phi}$ and $\tilde{\phi}_{m j}=\phi_{m j} \eta_{j}$. Here we assumed the orthonormal condition of wave functions

$$
\begin{aligned}
& \sum_{j} \tilde{\phi}_{m j} \tilde{\phi}_{m^{\prime} j}^{*}=\sum_{j} \phi_{m j} \phi_{m^{\prime} j}^{*} \eta_{j}^{2}=\delta_{m m^{\prime}} \\
& \sum_{m} \tilde{\phi}_{m j} \tilde{\phi}_{m j^{\prime}}^{*}=\sum_{m} \phi_{m j} \phi_{m j^{\prime}}^{*} \eta_{j} \eta_{j}^{\prime}=\delta_{j j^{\prime}}
\end{aligned}
$$

and used an identity

$$
\varepsilon_{j_{1} j_{2} j_{3} j_{4}} \tilde{\phi}_{m_{1} j_{1}} \tilde{\phi}_{m_{2} j_{2}} \tilde{\phi}_{m_{3} j_{3}} \tilde{\phi}_{m_{4} j_{4}}=\varepsilon_{m_{1} m_{2} m_{3} m_{4}} \Delta
$$

From Eq.(3.8) and (3.9) it follows that

$$
\varepsilon_{j_{1} j_{2} j_{3} j_{4}} \tilde{\phi}_{m_{3} j_{3}} \tilde{\phi}_{m_{4} j_{4}}=\tilde{\phi}_{m_{1} j_{1}}^{*} \tilde{\phi}_{m_{2} j_{2}}^{*} \varepsilon_{m_{1} m_{2} m_{3} m_{4}} \Delta
$$

which leads to (3.7). Note that the quantum numbers $m_{3}, m_{4}$ on the r.h.s. of $(3.7)$ turn out to be different from the ones (fixed) $m_{1}, m_{2}$ on the l.h.s. of (3.5). It is due to the factor $\varepsilon_{m_{1} m_{2} m_{3} m_{4}}$, or the Pauli's principle. The consequence of (3.9) is ; this state is similar to the original one (l.h.s. of (3.4)) if we replace (i) $c^{\dagger}$ by $d^{\dagger}$, (ii) $\sigma$ by $-\sigma$, (iii) $\phi_{m j}$ by $\tilde{\phi}_{\bar{m} j}^{*}=\phi_{\bar{m} j}^{*} \eta_{j}$, where $\bar{m}$ is the coset of $m$, namely a combination which is completely different from the original set of quantum number $m$. Having 
in mind the fact that the hamiltonian is symmetric under $\mathrm{p}$-h transformation, the original Green's function of spin $\sigma$ based on the wave function $\phi_{m j}$ is transformed to that of spin $-\sigma$ based on $\tilde{\phi}_{\bar{m} j}^{*}$.

\section{3-2 dual choice}

Consider a set of wave function $\left\{\phi_{m j}\right\}$ which defines (3.6) of single particle basis. We learned in the previous sub-section that the corresponding hole basis is

$\left\{\phi_{\bar{m} j}^{*} \eta_{j}\right\}$. When the set $\left\{\phi_{\bar{m} j} \eta_{j}\right\}$ coincides with the set $\left\{\phi_{m j}\right\}$, that is, when the hole basis wave function in (3.9) is simply complex conjugate of the particle basis wave function, we call the choice of the wave functions $\left\{\phi_{m j}\right\}$ "dual choice". Let us take the previous example of the one dimensional 4-site case. We can take $\phi_{m j}$ as

$$
\phi_{1 j}=1 \quad \phi_{2 j}=C \exp (i 2 \pi n / 4)
$$

where $n$ is an integer standing for the location $j$, and $C$ is a normalization constant. Then $\phi_{\bar{m} j}$ can be taken as

$$
\phi_{\overline{1} j}=C \exp (-i 2 \pi n / 4) \quad \phi_{\overline{2} j}=C \exp (i 4 \pi n / 4)
$$

We see that

$$
\phi_{1 j}^{*} \eta_{j}=\phi_{2 j}^{*} \quad \phi_{2 j}^{*} \eta_{j}=\phi_{1 j}^{*}
$$

and it shows the choice (3.11) is a "dual choice".

\section{3-3 sum rule}

Since the hamiltonian is invariant under the $\mathrm{p}$-h transformation, we drop for simplicity the Boltzmann factor $\exp (-\beta H)$ in the following arguments. Due to 
the dual choice of wave function, matrix element of $c$ 's is transformed to that of $d$ 's with the same set of wave functions except for complex conjugation.

$$
\begin{aligned}
& \left\langle 0\left|c_{m_{2} \sigma} c_{m_{1} \sigma} c_{m_{1} \sigma}^{\dagger} c_{m_{2} \sigma}^{\dagger}\right| 0\right\rangle \\
& =\sum_{j_{1} j_{2} j_{1}^{\prime} j_{2}^{\prime}}\left\langle\tilde{0}\left|d_{j_{2}^{\prime}-\sigma} d_{j_{1}^{\prime}-\sigma} \phi_{m_{1} j_{1}^{\prime}} \phi_{m_{2} j_{2}^{\prime}} \phi_{m_{1} j_{1}}^{*} \phi_{m_{2} j_{2}}^{*} d_{j_{1}-\sigma}^{\dagger} d_{j_{2}-\sigma}^{\dagger}\right| \tilde{0}\right\rangle
\end{aligned}
$$

The Green's function is changed to

$$
\begin{aligned}
G_{\sigma i j}(\phi) & =\left\langle 0\left|c_{m_{2} \sigma} c_{m_{1} \sigma} c_{i \sigma} c_{j \sigma}^{\dagger} c_{m_{1} \sigma}^{\dagger} c_{m_{2} \sigma}^{\dagger}\right| 0\right\rangle \\
& =\left\langle\tilde{0}\left|d_{j_{2}^{\prime}-\sigma} d_{j_{1}^{\prime}-\sigma} \phi_{m_{1} j_{1}^{\prime}} \phi_{m_{2} j_{2}^{\prime}} \eta_{i} d_{i-\sigma}^{\dagger} \eta_{j} d_{j-\sigma} \phi_{m_{1} j_{1}}^{*} \phi_{m_{2} j_{2}}^{*} d_{j_{1}-\sigma}^{\dagger} d_{j_{2}-\sigma}^{\dagger}\right| \tilde{0}\right\rangle \\
& =\delta_{i j}-\eta_{i} \eta_{j} G_{-\sigma j i}\left(\phi^{*}\right) \\
& =\delta_{i j}-\eta_{i} \eta_{j} G_{-\sigma j i}^{*}(\phi)
\end{aligned}
$$

where the reality of $G$ is used. We therefore have

$$
f_{i j}= \begin{cases}\delta_{i j}-g_{j i}^{*} & \text { for }|i-j|=\text { even } \\ g_{j i}^{*} & \text { for }|i-j|=\text { odd }\end{cases}
$$

where $f_{i j}$ and $g_{i j}$ denote the spin up and down Green's function in the configuration space.

\section{3-4 general case}

We now turn to general case where the lattice size $N$ is $N_{1} \times N_{2}\left(N_{1}\right.$ and $N_{2}$ stand for the number of lattice sites in the first and second direction) and the fermion number of each spin $M=N / 2(N=$ even $) . M$ fermion state is

$$
c_{m_{1}, \sigma}^{\dagger} c_{m_{2} \sigma}^{\dagger} \cdots c_{m_{M} \sigma}^{\dagger}|0\rangle=\sum_{j_{1} j_{2} \ldots j_{M}} \phi_{m_{1} j_{1}} \cdots \phi_{m_{M} j_{M}} c_{j_{1} \sigma}^{\dagger} c_{j_{2} \sigma}^{\dagger} \cdots c_{j_{M} \sigma}^{\dagger}|0\rangle .
$$


It changes to

$$
\begin{array}{r}
\sum_{j_{1} j_{2} \ldots j_{M}} \phi_{m_{1} j_{1}} \cdots \phi_{m_{M} j_{M}} \eta_{j_{1}} \eta_{j_{2}} \cdots \eta_{j_{M}} d_{j_{1}-\sigma} \cdots d_{j_{M}-\sigma} \\
\times \frac{1}{N !} \varepsilon_{i_{1} i_{2} \ldots i_{N}} d_{i_{1}-\sigma}^{\dagger} \cdots d_{i_{N}-\sigma}^{\dagger} d_{i_{1} \sigma}^{\dagger} \cdots d_{i_{N} \sigma}^{\dagger}|\tilde{0}\rangle
\end{array}
$$

Concerning the spin $-\sigma$ quantities, we have

$$
\begin{aligned}
& \frac{{ }_{N} C_{M} M !}{N !} \sum_{i_{1} \ldots i_{N}} \phi_{m_{1} i_{1}} \cdots \phi_{m_{M} i_{M}} \eta_{i_{1}} \eta_{i_{2}} \cdots \eta_{i_{M}} \\
& \times \varepsilon_{i_{1} i_{2} \ldots i_{M} i_{M+1} \ldots i_{N}} d_{i_{M+1}}^{\dagger} \cdots d_{i_{N}}^{\dagger}|\tilde{0}\rangle \\
& =\frac{{ }_{N} C_{M} M !}{N !} \Delta \sum_{\{j\} m_{M+1} \ldots m_{N}} \tilde{\phi}_{m_{M+1} j_{M+1}}^{*} \cdots \tilde{\phi}_{m_{N} j_{N}}^{*} \\
& \times \varepsilon_{m_{1} \ldots m_{M} m_{M+1} \ldots m_{N}} d_{j_{M+1}}^{\dagger} \cdots d_{j_{N}}^{\dagger}|\tilde{0}\rangle
\end{aligned}
$$

where $\Delta=\operatorname{det} \tilde{\phi}, \tilde{\phi}_{m j}=\phi_{m j} \eta_{j}$, and $m_{1} \ldots m_{M}$ are fixed. For dual choice, we have

$$
\tilde{\phi}_{m_{M+1} j_{M+1}}^{*} \cdots \tilde{\phi}_{m_{N} j_{N}}^{*}=\phi_{m_{1} j_{M+1}}^{*} \cdots \phi_{m_{M} j_{N}}^{*}
$$

So (3.20) tells us that the transformed wave functions can be given by the original wave functions. Only the difference from the original wave function is that they appear in complex conjugate form. We assume $|\Delta|=1$ because of the unitarity of the wave function. Along with the similar argument given in $\S 3-3$, we reach

$$
f_{i j}= \begin{cases}\delta_{i j}-g_{j i}^{*} & \text { even spacing } \\ g_{j i}^{*} & \text { odd spacing }\end{cases}
$$

3-5 $\chi S O$ vs. sum rule

In order to calculate the chiral spin order parameter $(\chi \mathrm{SO}){ }^{1}{ }^{1}$, six point function is necessary. Six point function with the same spin operators is decomposed into triple products of two point function (Green's function);

$$
\begin{aligned}
\left\langle c_{1}^{\dagger} c_{1} c_{2}^{\dagger} c_{2} c_{3}^{\dagger} c_{3}\right\rangle= & G_{11} G_{22} G_{33}-G_{12} G_{21} G_{33}+G_{12} G_{31} G_{23} \\
& -G_{11} G_{23} G_{32}+G_{13} G_{21} G_{32}-G_{13} G_{22} G_{31}
\end{aligned}
$$


The $\chi \mathrm{SO}$ is given by

$$
E_{123} \equiv\left\langle\vec{\sigma}_{1} \cdot\left(\vec{\sigma}_{2} \times \vec{\sigma}_{3}\right)\right\rangle=-2 i\left(P l_{123}-P l_{132}\right)
$$

where $\vec{\sigma}_{i}$ is Pauli matrices sitting at site $i$, and

$$
P l_{123}=\left\langle\chi_{12} \chi_{23} \chi_{31}\right\rangle=\left\langle c_{1 \sigma}^{\dagger} c_{2 \sigma} c_{2 \sigma^{\prime}}^{\dagger} c_{3 \sigma^{\prime}} c_{3 \sigma^{\prime \prime}}^{\dagger} c_{1 \sigma^{\prime \prime}}\right\rangle
$$

As is shown in the appendix, $\left(P l_{123}-P l_{132}\right)$ is purely real provided that the sum rule (3.21) holds. So a consequence of the sum rule is that the real part of the $\chi \mathrm{SO}$

$$
\operatorname{Re} E_{123}=2 \operatorname{Im}\left(P l_{123}-P l_{132}\right)
$$

becomes zero.

This holds only for the half-filling case and does not tell anything about doped cases. In the following section we will explicitly see the above properties by making numerical simulations.

\section{$\S 4$. Monte Carlo simulations}

In the present paper we take $4 \times 4$ spatial lattice, and the Trotter size $L$ is taken to be 100 through 200 , so that $\Delta \tau$ is sufficiently small $(\Delta \tau \leq 0.1)$. The hopping parameter $t$ is kept to be 1 , and $U$ is taken to be 4.0. The wave function $\left\{\phi_{m i}\right\}$ is on the free particle basis in the complex form $\exp \left(i \frac{2 \pi}{4} \mathbf{k} \cdot \mathbf{n}\right)$. We choose particle states with momentum $\mathbf{k}$ so as to fill the energy states in order from the lowest to higher ones. We make a dual choice of section 3. For half-filling, the lower-half energy states are occupied by $\left\{\phi_{m i}\right\}$ and the dual counterparts $\left\{\psi_{m i}\right\}$ correspond to the 
upper-half. Setting $\left\{\phi_{m i}\right\}=\exp [i 2 \pi \mathbf{k} \cdot \mathbf{n} / 4]$, our choice is $\mathbf{k}=\left(k_{1}, k_{2}\right)=(0,0)$ for $\phi_{m}=\phi_{1},(1,0)$ for $\phi_{2},(-1,0)$ for $\phi_{3},(0,1)$ for $\phi_{4},(0,-1)$ for $\phi_{5},(1,1)$ for $\phi_{6}$ and $(1,-1)$ for $\phi_{7}$. As for $\phi_{8}$, we choose a linear combination of the two states $(2,0)$ and $(0,2)$. Dual counterparts are chosen in such a way that $\left(k_{1}, k_{2}\right)=(2,2)$ for $\psi_{1},(-1,2)$ for $\psi_{2},(1,2)$ for $\psi_{3},(2,-1)$ for $\psi_{4},(2,1)$ for $\psi_{5},(-1,-1)$ for $\psi_{6}$, $(-1,1)$ for $\psi_{7}$ and $\psi_{8}=\phi_{8}$. In Fig.1, we show them in the momentum space. For spin up and down sectors we take the same wave functions $\left\{\phi_{m i}\right\}$, and $m$ runs from 1 to $M_{f}$. We performed from 3000 to 18000 sweeps depending on $M_{f}$ (the number of fermions with each spin $\sigma(2.11)$ ) and $\beta$. The statistical errors are estimated by use of the block method with each block size being 500 to 1000 after 1000 warming-up sweeps.

\section{1 half filled case $\left(M_{f}=8\right)$}

For the half filled case, no sign problem is involved, since the average of phase is purely real and is unity for any configurations occurred in the algorithm. We define symmetrized two point functions

$$
f_{\{i j\}} \equiv \frac{1}{2}\left(f_{i j}+f_{j i}\right) \quad g_{\{i j\}} \equiv \frac{1}{2}\left(g_{i j}+g_{j i}\right)
$$

Spin average of the symmetrized functions satisfy

$$
\begin{aligned}
& \operatorname{Re}\left(f_{\{i j\}}+g_{\{i j\}}\right)=0 \quad \text { for }|i-j|=\text { even } \neq 0 \\
& \operatorname{Re}\left(f_{\{i i\}}+g_{\{i i\}}\right)=1.0 \\
& \operatorname{Im}\left(f_{\{i j\}}+g_{\{i j\}}\right)=0 \quad \text { for }|i-j|=\text { odd }
\end{aligned}
$$

In Fig.2a, we show results of the Green's function, and compare with those of the Lanczos's method. ${ }^{15,16)}$ The average values of the real part for even spacings 
$(|i-j| \neq 0)$ are exactly zero in agreement with the argument in section 3 . Fig.2b is spin-spin correlations. It shows clear antiferromagnetic correlations and agrees very well with the Lanczos's one. The numerical figures for the Green's functions and the spin-spin correlations are listed in Table I.

The real part of $\chi \mathrm{SO}$ turns out exactly vanishing as discussed in the previous section. We looked also at the imaginary part, and it is consistent with zero within error.

\section{2 sign problem}

For simulations of doped cases one is confronted with the sign problem. In our case, for the complex trial wave function, $W$ is generally complex, although $\rho(\beta ; \Phi)$ (2.3) is real. As stated in section 2, one generates a sequence of configurations with a probability, ${ }^{23)}$

$$
P_{+}(s)=\frac{|W(s)|}{\rho_{+}}
$$

where

$$
\rho_{+}=\sum_{s}|W(s)|
$$

The average of the phase of $W$, which appears in $(2.22)$, is then given by

$$
<e^{i \theta_{w}}>_{+}=\rho / \rho_{+}
$$

For large $\beta, \rho$ is dominated by the ground state $\left|\psi_{0}\right\rangle$ with energy $E_{0}$

$$
\rho \simeq \exp \left(-\beta E_{0}\right)\left|<\psi_{0}\right| \Phi>\left.\right|^{2}
$$


while $\rho_{+}$looks like

$$
\rho_{+} \simeq \exp \left(-\beta E_{+}\right)\left|<\psi_{+0}\right| \Phi>\left.\right|^{2}
$$

where $E_{+}$and $\psi_{+0}$ are analogue of $E_{0}$ and $\psi_{0}$ for $\rho_{+}$, respectively. Therefore the average of the phase is real, and behaves in a similar manner to that of the sign in the real trial wave function case;

$$
\left\langle e^{i \theta_{w}}\right\rangle_{+}=\rho / \rho_{+} \simeq \exp (-\beta \Delta E)
$$

where $\Delta E \equiv E_{0}-E_{+}$. For $\Delta E=0$, the average of sign converges to some finite value. In this case, one has no difficulty with the phase, and one may neglect the phase for evaluating the average of physical quantities. For $\Delta E \neq 0$, on the other hand, we encounter so called the sign problem, that is, $\left\langle e^{i \theta_{w}}\right\rangle$ becomes vanishing in our case. It apparently gives meaningless result for the average of any physical operator (2.22) and anticipates, at the same time, large errors. If, however, the numerator in (2.22) behaves in a exponential manner similarly to the denominator, it is still expected that the average may converge to some finite value, and its errors could be tamable. This depends upon the fillingness and operators to be calculated. In the following subsections we show the results concerned with this issue for $M_{f}=7$ and $M_{f}=5$ (closed shell).

\subsection{Hole doped cases $M_{f}=7$}

The average of the real part of the phase is shown in Fig.3 and Table II. In the region we have calculated $(\beta=2.0$ to 5.0 ), an exponential behavior is clearly observed. The $\Delta E$ read from the slope is $1.01(5)$. This value is somewhat larger 
than the one obtained by Loh et al. ${ }^{23)}$ The average $<O>_{+}$with the measure (2.21) for Green's functions $\left(O=G_{i j}\right)$ and for spin correlations are determined in a high precision, while the real part of the $O=\chi \mathrm{SO}$ parameter is somewhat noisy and looks unstable as $\beta$ varies. This difference of the fluctuations in $\langle O\rangle_{+}$ results in the difference of weighted averages (2.22) among the Green's function, spin-spin correlations and the $\chi \mathrm{SO}$.

According to the formula (2.22), the average of observables is proportional to the product of observables and the phase in the numerator. We have found that due to the steadiness of behaviors of the Green's function $G_{i j}$ and spin-spin correlations with respect to a variation of $\beta$, their product shows an exponential fall-off with almost the same slope as the denominator $\left\langle e^{i \theta_{w}}\right\rangle_{+}$. On the other hand, the product of $\chi \mathrm{SO}$ and phase does not show such behavior. Fig.4a and $4 \mathrm{~b}$ show the real and the imaginary parts of the $\chi \mathrm{SO}$ as a function of $\beta$. We see that for small $\beta$ ( $\beta=2.0$ and 3.0, for example), the real part has some positive value $^{15)}$ within error, but becomes unfortunately too noisy to extrapolate to the larger $\beta$ region (see Table II). We should note that the choice of the wave function in Fig.1 explicitly breaks the parity. This could be the reason why the $\chi \mathrm{SO}$ may be non-vanishing for small $\beta$. It is then important to study how the operator behaves in the large $\beta$ limit. Since the spontaneous symmetry breaking never happens in a finite volume in the strict sense ${ }^{24)}$ the $\chi \mathrm{SO}$ goes to zero as $\beta$ increases. As in the case of an introduction of an external source to break explicitly a symmetry, we must study finite size effects how the operator becomes vanishing. This is under investigation. 
We have got better results as to the Green's function and the spin-spin correlations. Fig.5 compares them with the Lanczos method. According to Parola et al., ${ }^{15,16)}$ the ground states are found to be threefold degenerate; one is the d-wave state of momentum $(0,0)$ and the others are a pair of $(0, \pi)$ and $(\pi, 0)$. Both the states show slightly different behavior for the density distribution. The Monte Carlo results are in good agreement with that for $(0,0)$. Spin-spin correlations are shown in Fig.6. The data used for drawing the figures are listed in Table I.

\subsection{Hole doped cases $M_{f}=5$}

The case $M_{f}=5$ makes a "closed shell" in the single free particle distribution in the momentum space. The sign problem is very much mild compared to the $M_{f}=7$ case. The average of the real part of the phase is shown in Fig. 7 and Table II. One sees an exponential fall-off behavior for a wide range of $\beta$ with a decay rate being quite small compared to the $M_{f}=7$ case; $\Delta E=0.0018(3)$. We see then that $\Delta E$ depends much on the fillingness. The unweighted Green's function as a function of $\beta$ are very stable and gets very small errors. These facts lead us to expect that the weighted average is approximately given by the unweighted one. In other words, a correction to the unweighted average is quite small. In Fig.8, we compare them with the ones obtained by the Lanczos method. We see a very good agreement. The spin-spin correlations ( Fig.9.) are also in very good agreement with the exact numerical calculations. ${ }^{16)}$ The numerical figures are listed in Table Ifor the Green's function and the spin-spin correlations. As for the behavior of $\chi$, both the real and imaginary parts are consistent with zero within error. 


\section{$\S 5$. Summary and Discussion}

We performed Monte Carlo calculations of the two dimensional Hubbard model by use of complex wave functions as a trial state. As to the single particle density operators, or the Green's functions and spin-spin correlations, we have seen good agreements with the Lanczos' results for half-filled and doped cases. Encouraged by this fact, we tried to measure the $\chi \mathrm{SO}$. For half filled case, without numerical calculations, we have seen that it is vanishing based upon the particle-hole transformation. Upon doping, the phase problem is a main obstacle. For $M_{f}=7$, the problem is rather serious, and we are not able to extrapolate to large enough values of $\beta$, though the real part of the $\chi \mathrm{SO}$ looks nonvanishing at small $\beta$. In order to clarify it, we need to study finite size effect to see how this operator reaches zero, since no spontaneous symmetry breaking occurs in finite volume in the strict sense. This issue is under investigation. For $M_{f}=5$, the phase problem is very mild. In this case, the real part of the $\chi \mathrm{SO}$ is consistent with zero independent of values of $\beta$. As to the average of plaquettes, which is a product of four links $\left\langle\chi_{12} \chi_{23} \chi_{34} \chi_{41}\right\rangle$, we will report in the forthcoming paper.

\section{ACKNOWLEDGMENTS}

We are grateful to Dr. N. Hatano for discussions concerning the measurement of the $\chi \mathrm{SO}$. We appreciate the collaboration of K. Yamaji in the early stage of this work. We are very much indebted to the Illinois University, where the preliminary calculations were performed, and to the RCNP, where the main calculations have been done. 


\section{References}

1) X.G. Wen, F. Wilczek and A. Zee, Phys. Rev. B39(1989), 11413 .

2) P.W. Anderson, Science235(1987), 1196.

3) G.Baskaran and P.W.Anderson, Phys. Rev. B37(1988), 580 .

4) I. Affleck and B. Marston, Phys. Rev.B37 (1988), 3774.

5) A.N. Redlich, Rhys. Rev. Lett.52(1984), 18.; Rhys. Rev. D29(1984), 2366.

6) H. So, Prog. Theor. Phys.73(1985), 528.; 74(1985), 585.

7) K. Ishikawa, Phys. Rev. Lett.53(1984), 1615.

8) E. Fradkin and F.A. Shaposnik, Phys. Rev. Lett.66(1991), 276.

9) J.E. Hirsch, Phys. Rev.B28(1983), 4059.

10) M. Imada and Y. Hatsugai, J. Phys. Soc. Jpn. 58(1989), 3752.

11) J. Hubbard, Phys. Rev. Lett.3(1959), 77.

12) G. Kotliar , Phys. Rev. B37(1988), 3664.

13) E.T. Tomboulis, Phys. Rev. Lett.68(1992), 3100.

14) N. Furukawa and M. Imada, J. Phys. Soc. Jpn60(1991), 3669.

15) A. Parola, S.Sorella, M.Parrinello and E.Tosatti, Phys. Rev.B43(1991), 6190.

16) A. Parola, S.Sorella, S.Baroni, M.Parrinello and E.Tosatti, Int. J. Mod. Phys.B3(1989), 1865.

17) E. Dagotto, A.Moreo, F.Ortolani, D.Poilblanc, J.Riera and D.Scalapino, Phys. Rev. B45(1992), 10741.

18) J.E. Hirsch, J. of Stat. Phys.43(1986), 841. 
19) J.E. Hirsch, Phys. Rev.B31(1985), 4403.

20) J.E. Hirsch, Phys. Rev. Lett.51(1983), 1900.

21) H.F. Trotter, Proc. Am. Math. Soc.10(1959), 545.

22) M. Suzuki, Prog. Theor. Phys.56(1976), 1454.

23) E.Y. Loh Jr., F.E.Gubernatis, R.T.Scalettar, S.R.White, D.J.Scalapino and R.L.Sugar, Phys. Rev.B41(1990), 9301.

24) N. Hatano and M. Suzuki , J. Phys. Soc. Jpn.62(1993), 847. 


\section{TABLE CAPTIONS}

Table I The average values and the statistical errors of the single particle density operators (spin averaged symmetrical Green's function) $G_{i}$ and the spin-spin correlations $S_{i}$ for various fillings. Suffix $i$ stands for the locations of the sites along the path as depicted in the inset of Fig.2a. The values for $M_{f}=8, M_{f}=$ 7 and $M_{f}=5$ are shown at $\beta=5.0, \beta=4.0$ and $\beta=20.0$, respectively. Note that $G_{0}=1.0$ for $M_{f}=8$.

Table II The real part of the average of the phase vs. $\beta$ for $M_{f}=7$ and $M_{f}=5$. The real and imaginary parts of the $\chi \mathrm{SO}$ is also listed for $\beta=7$. Meas stands for the number of measurements $(1 k=1000)$.

\section{FIGURE CAPTIONS}

Fig. 1 Illustration of the "Dual choice" of wave functions in the momentum space. $\left\{\phi_{m}\right\}$ and $\left\{\psi_{m}\right\}$ are shown (see text).

Fig. 2a Expectation value of single particle density operator, or spin averaged symmetrical Green's function $G_{i}$ for half-filling and $\beta=5.0$. The horizontal axis stands for the locations $i$ along the path shown in the inset. The first and third sites are odd, and the rests are even spacings. Circle is the results of the Monte Carlo calculations, while cross is the Lanczos' results. ${ }^{16)}$ Lines are drawn for the guide of eyes. 
Fig. 2b Spin-spin correlations $S_{i}$ indicate clear antiferromagnetic correlations for half filling and $\beta=5.0$. The horizontal axis stands for the same location $i$ as in Fig.2a. Monte Carlo calculations ( circle) are in good agreement with those of Lanczos' method (cross). ${ }^{16)}$ The errors lie within the symbols.

Fig. 3 Average of the real part of the phase of the Monte Carlo weights vs. $\beta$ for $M_{f}=7$.

Fig. 4a Real part of the $\chi \mathrm{SO}$ vs. $\beta$ for $M_{f}=7$.

Fig. 4b Imaginary part of the $\chi \mathrm{SO}$ vs. $\beta$ for $M_{f}=7$.

Fig. 5 Single particle density operator $G_{i}$ for $M_{f}=7$ and $\beta=4.0$. We compare the Monte Carlo results (circle) with the Lanczos' ones. ${ }^{16)}$ According to Parola et. al., ${ }^{16)}$ the degenerate ground states (see text) show different behavior. Monte Carlo results agree well with the behavior of the $(0,0)$ state (cross). The symbol (square) stands for the behavior of $(0, \pi)$ and $(\pi, 0)$ state.

Fig. 6 Spin-spin correlations $S_{i}$ for $M_{f}=7$ and $\beta=4.0$. Monte Carlo results (circle)

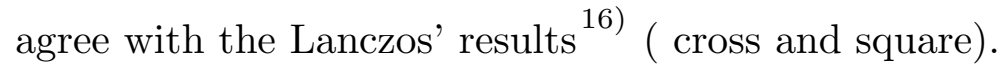

Fig. 7 The real part of the phase of the Monte Carlo weights vs. $\beta$ for $M_{f}=5$. An exponential fall-off behavior is seen in the region where $\beta \geq 10.0$.

Fig. 8 Single particle density operator $G_{i}$ for $M_{f}=5$ and $\beta=20.0$. The Monte Carlo results (circle) are in very good agreement with those of Lanczos' method ${ }^{16)}$ (cross). The errors lie within the symbols.

Fig. 9 Spin-spin correlations $S_{i}$ (circle) for $M_{f}=5$ and $\beta=20.0$. Comparison with the Lanczos' results ${ }^{16)}$ (cross). The errors lie within the symbols. 


\section{APPENDIX}

In this appendix, we show that $\left(P l_{123}-P l_{132}\right)$ appearing in $(3.23)$ is pure real. $\chi \mathrm{SO}$ is expressed by two through six point functions. Four point functions are

$$
\begin{aligned}
& \langle\overline{1} 1 \overline{2} 2\rangle_{\sigma} \equiv\left\langle c_{1 \sigma}^{\dagger} c_{1 \sigma} c_{2 \sigma}^{\dagger} c_{2 \sigma}\right\rangle=G_{11}^{(\sigma)} G_{22}^{(\sigma)}-G_{12}^{(\sigma)} G_{21}^{(\sigma)} \\
& \langle\overline{1} 2 \overline{2} 3\rangle_{\sigma} \equiv\left\langle c_{1 \sigma}^{\dagger} c_{2 \sigma} c_{2 \sigma}^{\dagger} c_{3 \sigma}\right\rangle=G_{31}^{(\sigma)}-G_{31}^{(\sigma)} G_{22}^{(\sigma)}+G_{32}^{(\sigma)} G_{21}^{(\sigma)}
\end{aligned}
$$

where $G_{i j}^{(\sigma)}=\left\langle c_{j \sigma}^{\dagger} c_{i \sigma}\right\rangle$ for spin $\sigma .\left(P l_{123}-P l_{132}\right)$ in $(3.23)$

$$
P l_{123}-P l_{132}=\sum_{\sigma, \sigma^{\prime}, \sigma^{\prime \prime}}\left\{\left\langle c_{1 \sigma}^{\dagger} c_{2 \sigma} c_{2 \sigma^{\prime}}^{\dagger} c_{3 \sigma^{\prime}} c_{3 \sigma^{\prime \prime}}^{\dagger} c_{1 \sigma^{\prime \prime}}\right\rangle-\left\langle c_{1 \sigma}^{\dagger} c_{3 \sigma} c_{3 \sigma^{\prime}}^{\dagger} c_{2 \sigma^{\prime}} c_{2 \sigma^{\prime \prime}}^{\dagger} c_{1 \sigma^{\prime \prime}}\right\rangle\right\}
$$

is written as

$$
\begin{aligned}
P l_{123}-P l_{132} & =\langle\overline{1} 1 \overline{2} 2 \overline{3} 3\rangle_{\uparrow}-\langle\overline{1} 1 \overline{3} 3 \overline{2} 2\rangle_{\uparrow}+\langle\overline{1} 2 \overline{2} 3\rangle_{\uparrow}\langle\overline{3} 1\rangle_{\downarrow}-\langle\overline{1} 3 \overline{3} 2\rangle_{\uparrow}\langle\overline{2} 1\rangle_{\downarrow} \\
& +\langle\overline{1} 2 \overline{3} 1\rangle_{\uparrow}\langle\overline{2} 3\rangle_{\downarrow}-\langle\overline{1} 3 \overline{2} 1\rangle_{\uparrow}\langle\overline{3} 2\rangle_{\downarrow}+\langle\overline{2} 3 \overline{3} 1\rangle_{\uparrow}\langle\overline{1} 2\rangle_{\downarrow}-\langle\overline{1} 3\rangle_{\uparrow}\langle\overline{3} 2 \overline{2} 1\rangle_{\downarrow} \\
& +\langle\overline{1} 2\rangle_{\uparrow}\langle\overline{2} 3 \overline{3} 1\rangle_{\downarrow}-\langle\overline{1} 3\rangle_{\uparrow}\langle\overline{3} 2 \overline{2} 1\rangle_{\downarrow}+\langle\overline{2} 3\rangle_{\uparrow}\langle\overline{1} 2 \overline{3} 1\rangle_{\downarrow}-\langle\overline{3} 2\rangle_{\uparrow}\langle\overline{1} 3 \overline{2} 1\rangle_{\downarrow} \\
& +\langle\overline{3} 1\rangle_{\uparrow}\langle\overline{1} 2 \overline{2} 3\rangle_{\downarrow}-\langle\overline{2} 1\rangle_{\uparrow}\langle\overline{1} 3 \overline{3} 2\rangle_{\downarrow}+\langle\overline{1} 1 \overline{2} 2 \overline{3} 3\rangle_{\downarrow}-\langle\overline{1} 1 \overline{3} 3 \overline{2} 2\rangle_{\downarrow} \\
& =-f_{22} f_{31} g_{13}+f_{22} f_{13} g_{31}+g_{22} f_{31} g_{13}-g_{22} f_{13} g_{31} \\
& +\left(\text { terms without } \mathrm{f}_{22} \text { or } \mathrm{g}_{22}\right)
\end{aligned}
$$

where we used the notation $f_{i j} \equiv G_{i j}^{(\uparrow)}$ and $g_{i j} \equiv G_{i j}^{(\downarrow)}$. The sum rule (3.21) and (A.3) lead to

$$
\begin{aligned}
P l_{123}-P l_{132} & \\
& =\left(g_{13}^{*} g_{13}-g_{31}^{*} g_{31}\right)-\left(g_{22}^{*}+g_{22}\right)\left(g_{13}^{*} g_{13}-g_{31}^{*} g_{31}\right) \\
& +\left(\text { terms without } \mathrm{f}_{22} \text { or } \mathrm{g}_{22}\right) \\
& =\text { pure real }+\left(\text { terms without } \mathrm{f}_{22} \text { or } \mathrm{g}_{22}\right)
\end{aligned}
$$


The terms without $f_{22}$ or $g_{22}$ in (A.4) can also be rewritten by the sum rule (3.21) in the form as sum of terms $\alpha+\alpha^{*}$ and $\beta \beta^{*}$, where $\alpha$ and $\beta$ are complex numbers. 
This figure "fig1-1.png" is available in "png" format from: http://arxiv.org/ps/hep-lat/9312073v1 
This figure "fig2-1.png" is available in "png" format from: http://arxiv.org/ps/hep-lat/9312073v1 
This figure "fig1-2.png" is available in "png" format from: http://arxiv.org/ps/hep-lat/9312073v1 
This figure "fig2-2.png" is available in "png" format from: http://arxiv.org/ps/hep-lat/9312073v1 
This figure "fig2-3.png" is available in "png" format from: http://arxiv.org/ps/hep-lat/9312073v1 